\title{
Lewis acid/base character and crystallisation properties of poly(butylene terephthalate)
}

\author{
José M.R.C.A. Santos*, James T. Guthrie \\ Department of Colour and Polymer Science, School of Chemistry, The University of Leeds, Woodhouse Lane, Leeds, West Yorkshire LS2 9JT, United Kingdom
}

\section{A R T I C L E I N F O}

\section{Article history:}

Received 11 September 2014

Received in revised form

12 December 2014

Accepted 15 December 2014

Available online 29 December 2014

\section{Keywords:}

Inverse gas chromatography

Poly(butylene terephthalate)

Lewis acid/base properties

Crystallisation

\begin{abstract}
A B S T R A C T
Two grades of poly(butylene terephthalate) were analysed by means of inverse gas chromatography (IGC) and the results correlated with the respective crystallisation properties. The following parameters were determined by IGC: the dispersive component of the surface tension, the enthalpy and the entropy of adsorption of selected polar and apolar probes, and the Lewis acidity and basicity constants, $K_{a}$ and $K_{b}$ respectively. The interpretation of the values determined for $K_{a}$ and $K_{b}$ is in agreement with the FTIR spectra relating to the carboxyl end-group and the hydroxyl end-group concentrations in these polymers. The differences in the molecular weight values and in the end-group type and concentration, between the two grades of PBT, do not cause differences in the crystallisation activation energy. This observation suggests that there is a leading contribution of the Lewis basic sites to the crystallisation activation energy of the grades of PBT that were analysed. However, the lower value of $K_{a}$ and the greater molar mass of one of the PBT grades lead to a corresponding lower crystallisation degree.
\end{abstract}

(C) 2014 Elsevier B.V. All rights reserved.

\section{Introduction}

Due to their semi-crystalline character and high melting temperatures, poly(butylene terephthalate), PBT, and poly(ethylene terephthalate), PET, are the more widely used thermoplastic polyesters. Common applications of PET include fibre spinning, blow moulding of bottles, injection moulding of engineering parts and thermoforming of trays. PET is, nevertheless, characterised by low crystallisation rates. This has limited its application in injection moulding appliances. To overcome this drawback, fast crystallising grades have been developed that contain nucleating agents (e.g. $\mathrm{BaSO}_{4}, \mathrm{ZnPO}_{4}$ and $\mathrm{Sb}_{2} \mathrm{O}_{3}$ ) to increase the non-isothermal crystallisation temperature and reduce the size of the spherulites, and/or contain other polymers. PBT, on the other hand, exhibits a significantly faster crystallisation rate. The realised shorter moulding cycles and the lower viscosity under appropriate conditions have resulted in a wider use of PBT than PET for moulding applications.

PBT is actually one of the faster crystallising polymers [1-5]. The chemical nature of the repeat unit is shown in Fig. 1. The high crystallisation rate is a consequence of the considerable mobility that

\footnotetext{
* Corresponding author. Present address: Polytechnic Institute of Bragança, Campus Sta. Apolónia, 5300-253 Bragança, Portugal. Tel.: +351 273330 832; fax: +351273325 405 .

E-mail addresses: josesantos@ipb.pt, josemrcasantos@gmail.com (J.M.R.C.A. Santos)
}

is provided by the butylene unit in the chain. Usually, pure PBT has a crystallinity extent that is in the range of $30-40 \%$. The enthalpy of fusion, $\Delta H_{\mathrm{f}}{ }^{\circ}$, of the $100 \%$ crystalline PBT is $142 \mathrm{~J} / \mathrm{g}[4,6-8]$. The melting temperature $T_{\mathrm{m}}$ is about $225^{\circ} \mathrm{C}$ and the crystallisation temperature, $T_{\mathrm{c}}$, is approximately $180^{\circ} \mathrm{C}$ [9]. The value of the glass transition temperature, $T_{\mathrm{g}}$, of the semicrystalline state occurs at about $40^{\circ} \mathrm{C}$. The completely amorphous state of pure PBT is difficult to produce due to the high crystallisation rate of this polymer. Nevertheless, a value of $-25^{\circ} \mathrm{C}$ has been determined for the $T_{\mathrm{g}}$ of $100 \%$ amorphous PBT $[7,10]$.

Because of its semi-crystalline nature, PBT offers considerable chemical/solvent resistance but low dimensional stability, low ductility, low glass transition temperature and low Izod impact strength. Consequently, core-shell type elastomers are most frequently added to PBT to improve its toughness. These blends show low melt viscosity and, thus, are easy to process. Moreover, the blends have good mechanical properties and electrical properties, excellent solvent resistance, and good hydrolytic stability. Blends of PBT with elastomers and with other polymers have been used for the injection moulding of exterior automotive parts such as mirror housings and bumpers, and are often designed to replace metals in specific applications.

Furthermore, in order to take full advantage of the useful properties of PBT, and to overcome the aforementioned limitations, PBT is usually combined with polymers such as polycarbonates, PC, and PET (e.g. Xenoy ${ }^{\circledR}$ and Valox ${ }^{\circledR}$ from SABIC Innovative Plastics, Makroblend ${ }^{\circledR}$ from Bayer, and Pocan ${ }^{\circledR}$ from Lanxess, Sabre ${ }^{\circledR}$ 


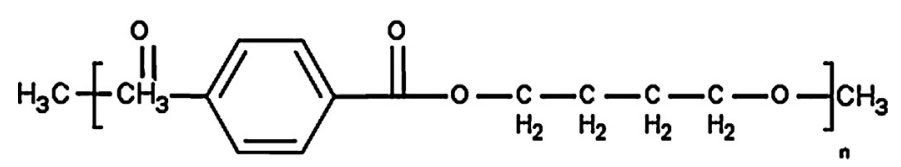

Fig. 1. Repeating unit in PBT.

Table 1

Weight average molecular weight $\left(M_{w}\right)$, molecular weight distribution $(D)$ and number-average molecular weight $\left(M_{n}\right)$, carboxyl end-group concentration and hydroxyl end-group concentration, for PBT A and PBT B.

\begin{tabular}{lllll}
\hline & $M_{w}\left(\mathrm{~g} \mathrm{~mol}^{-1}\right)$ & $D$ & $M_{n}\left(\mathrm{~g} \mathrm{~mol}^{-1}\right)$ & $-\mathrm{COOH}(\mu \mathrm{eq} / \mathrm{g})$ \\
\hline PBT A & 108,500 & 3.18 & 34,100 & 48 \\
PBT B & 46,000 & 2.70 & 17,000 & 29 \\
\hline
\end{tabular}

1600 from Dow Chemical Company, Stapron ${ }^{\circledR}$ E from DSM and Ultrablend ${ }^{\circledR}$ KR from BASF). In the polymer blends thus obtained, control over the crystalline properties of the PBT is necessary. During processing at elevated temperatures, hydrolytic, thermal, and oxidative degradation of PBT can occur with the formation of new carboxylic acid end-groups, along with the reduction in the molecular weight and alteration of the molecular weight distribution. These effects result in higher crystallisation rates and crystallisation extents $[4,5,8,11]$. In the particular case of PBT/PC blends, the formation of a PC-PBT copolyester results in a decrease in the molecular weight of the PC and of the PBT, in a reduction in the degree of crystallinity and in the crystallisation temperature $[2,6,7,12-14]$.

The growing awareness of the importance of solid surfaces, interfaces and interphases in determining the useful properties of polymeric compositions, has led to the development of inverse gas chromatography (IGC) as a valuable technique for evaluating the potential for interaction of different components of polymer blends and composites and multicomponent polymeric systems in general. Due to its applicability in determining the surface properties of solids in varied forms such as films, fibres and powders of both crystalline and amorphous structures, IGC has been extensively used for surface characterisation. The applicability of IGC in measurements of physicochemical properties of various materials has been thoroughly described in the literature [15-18]. Data obtained from IGC experiments may, in favourable cases, correlate directly with observed performance criteria, such as colour development, gloss, rheological properties, adhesion and mechanical properties [19-21].

Two papers have been published that dealt with the analysis of the thermodynamic properties of PBT (surface free energy, surface Lewis acidity and surface Lewis basicity), one that concerns a study carried out on a particular grade of PBT and a second that relates to surface tension studies on PBT, where the dispersive component of the surface tension was quantified using contact angle measurements $[22,23]$. The specific component of the surface free energy (without quantifying the contribution of the Lewis acidic sites and of the Lewis basic sites) was determined thereof.

Differences in end-group "concentrations" alongside with differences in the average molecular weight of PBT permit the tailoring of the contribution of the PBT phase towards the properties of the PBT-based blends, namely the viscosity and the crystallinity. Thus, they influence the processability and mechanical properties of these polymeric systems. For this study, IGC at infinite dilution was used as a tool by which to assess the differences between the surface Lewis acidic/basic properties of two grades of PBT, differentiated by their varying end-group "concentration". Some of the effects that the differences in end-group "concentration" and molecular weight have on the crystallisation properties were also examined.
Table 2

Relevant characteristics of commonly used IGC probes.

\begin{tabular}{llcc}
\hline Probe molecule & $\begin{array}{l}a\left(\gamma_{l}^{d}\right)^{0.5} \\
\left.\left(\mathrm{~cm}^{2}(\mathrm{~mJ} \mathrm{~cm})^{-2}\right)^{0.5}\right)\end{array}$ & $D N(\mathrm{~kJ} / \mathrm{mol})$ & $A N^{*}(\mathrm{~kJ} / \mathrm{mol})$ \\
\hline n-Hexane & $2.21 \mathrm{E}-16$ & - & - \\
n-Heptane & $2.57 \mathrm{E}-16$ & - & - \\
n-Octane & $2.91 \mathrm{E}-16$ & - & - \\
n-Nonane & $3.29 \mathrm{E}-16$ & - & - \\
n-Decane & $3.63 \mathrm{E}-16$ & 84.42 & 2.10 \\
Tetrahydrofuran (THF) & $2.13 \mathrm{E}-16$ & 0.00 & 22.68 \\
Trichloromethane (TCM) & $2.24 \mathrm{E}-16$ & 0.00 & 16.38 \\
Dichloromethane (DCM) & $1.73 \mathrm{E}-16$ & 80.64 & 5.88 \\
Diethyl ether (DEE) & $1.82 \mathrm{E}-16$ & 71.40 & 10.50 \\
Acetone (Acet) & $1.65 \mathrm{E}-16$ & 71.82 & 6.30 \\
Ethyl acetate (EtAcet) & $1.95 \mathrm{E}-16$ & & \\
\hline
\end{tabular}

Another aspect of relevance to this study was the fact that the specific component of the enthalpy of adsorption of the polar probes on the surface of PBT A and on the surface of PBT B is endothermic.

\section{Material and methods}

\subsection{Materials}

Two grades of poly(butylene terephthalate), PBT A and PBT B, were kindly supplied by SABIC Innovative Plastics (formerly GE Plastics Europe), Bergen op Zoom, The Netherlands. The average molecular weight, $M_{w}$, molecular weight distribution, $D$, numberaverage molecular weight, $M_{n}$, carboxyl end-group concentration and hydroxyl end-group concentration of these poly(butylene terephthalate)s are presented in Table 1. The hydroxyl end-group and carboxyl end-group concentrations were determined using the $-\mathrm{OH}$ absorbance at $3550 \mathrm{~cm}^{-1}$ and the $-\mathrm{COOH}$ absorbance at $3290 \mathrm{~cm}^{-1}$, respectively. The values indicated in Table 1 were obtained from the supplier. The glass transition temperature and the melting temperature of both PBT A and PBT B, are $318 \mathrm{~K}$ and $503 \mathrm{~K}$, respectively (determined by DSC).

For the IGC analysis, analytical grade (Sigma-Aldrich Ltd.) probes were used without further purification. The apolar probes used were n-heptane, n-octane, n-nonane, n-decane, and the polar probes tetrahydrofuran (THF, basic probe), the amphoteric probes acetone (Acet) and diethyl ether (DEE), and the acidic probes chloroform (TCM) and dichloromethane (DCM). In Table 2 are summarised relevant properties of the probe molecules mentioned [17-21]. The chemicals used as probe molecules were obtained from Sigma-Aldrich Ltd., Poole, UK. Methane (Phase Separations Ltd., Deeside, UK) was used as a non-interacting reference probe and the carrier gas used was helium (99.999+\% purity, BOC Gases Ltd., Guildford, UK).

\subsection{Inverse gas chromatography}

\subsubsection{IGC data processing}

The main difference between conventional gas chromatography (GC) and IGC lies in the fact that the species of primary interest are not the volatile components injected but the material acting as the stationary phase, typically a powder, fibre or film. This material may be packed directly into the column, coated onto a suitable support or coated onto the walls of the column. This allows the investigation of the interactive nature via the degree of interaction with well-characterised volatile liquids/vapours ("probes"). Quantification of this interaction may be achieved by the determination of the retention time, $t_{r}$, for a given probe. In most uses, the quantity of probe vapour injected into the carrier gas is extremely small. Thus, the retention data relate to the thermodynamic interaction 
that occurs between polymer and the vapour when the polymer is highly concentrated, as in most practical situations. Furthermore, IGC experiments may be carried out over appreciable temperature ranges, so that the temperature dependence of thermodynamic interactions is no longer indeterminate.

IGC data processing was carried out according to methods described in the literature [15,24,28,32]. The retention times of apolar probe molecules and of polar probe molecules were determined at specific temperatures, and the values of the retention volume, the energy of adsorption, the enthalpy and entropy of adsorption (dispersive and specific components) of the probes, and of the surface Lewis acidity and basicity constants, $K_{a}$ and $K_{b}$, respectively, were computed.

In IGC, an inert carrier gas elutes a minute quantity of a probe molecule through a column that is packed with the material under study. Due to the interactions between the two phases, the probe molecules are retained for a certain time, $t_{r}$, which is used to calculate the net retention volume, $V_{n}$, according to Eq. (1):

$V_{n}=\left(t_{r}-t_{0}\right) F \cdot C \cdot J$

Here, $V_{n}$ is the retention volume, $t_{0}$ is the dead retention time of the marker probe, $F$ is the carrier gas flow rate, $C$ is a correction factor, allowing for the vapour pressure of the water at the temperature of the bubble flow metre used to determine the flow rate, and $J$ is the correction factor for gas compressibility. The retention time was determined using the geometric technique outlined by Conder and Young $[29,30]$.

Assuming that experiments take place at infinite dilution, the free energy of adsorption of the probe on the stationary phase surface per mole, $\Delta G$, can be determined from the retention volume, $V_{n}$, according to:

$\Delta G=-R T \ln \left(V_{n}\right)+C_{1}$

Here, $R$ is the ideal gas constant, $T$ is the absolute column temperature and $C_{1}$ is a constant, which depends upon the chromatographic column and the reference state [27]. Throughout the IGC studies, the standard deviation of the energy of adsorption values of the probe molecules was calculated as being typically below $5 \%$.

Considering that the dispersive and specific components, respectively $\Delta G^{d}$ and $\Delta G^{s}$, are additive, as suggested by Fowkes [31], Eq. (2) can be rewritten as:

$\Delta G^{d}+\Delta G^{s}=-R T \ln \left(V_{n}\right)+C_{1}$

The free energy of adsorption can be related to adhesion work, $W_{a}$, according to [27]:

$-\Delta G=N \cdot a \cdot W_{a}$

Here, $N$ is Avogadro's number and " $a$ " the cross-sectional area of the probe to be tested (Table 2).

If non polar components (such as n-alkanes) are used, only dispersive interactions occur and the adhesion work is given by:

$W_{a}=2\left(\gamma_{s}^{d} \gamma_{l}^{d}\right)^{1 / 2}$

Here, $\gamma_{s}^{d}$ and $\gamma_{l}^{d}$ are, respectively, the dispersive components of surface tension of the solid (stationary phase) and of the probemolecule.

Replacing Eqs. (4) and (5) in Eq. (2) leads to:

$2 N\left(\gamma_{s}^{d}\right)^{1 / 2} a\left(\gamma_{l}^{d}\right)^{1 / 2}+C_{1}=R T \ln \left(V_{n}\right)$

The slope of the straight line, referred to as the reference line, obtained by plotting $R T \ln \left(V_{n}\right)$ versus $2 a N\left(\gamma_{l}^{d}\right)^{1 / 2}$, for a homologous n-alkane series (Fig. 4), leads to the determination of $\gamma_{s}^{d}$ for a given temperature.
Acid-base characteristics of surfaces were determined by analysing the interaction of the polar probes with the solid surface and quantifying the deviation from the reference line, leading to the estimation of the specific free energy, $\Delta G^{S}$, as:

$-\Delta G^{S}=R T \ln \left(V_{n}\right)-R T \ln \left(V_{n_{\text {ref }}}\right)$

Here, $V_{n_{r e f}}$ is the retention volume established by the n-alkanes reference line (Eq. (1)), $V_{n}$ being now the retention volume of the polar probes. This calculation is also illustrated in Fig. 4.

The adhesion work between the polar probes tested and the solid surface, $W_{a}^{s}$, can be evaluated from the specific free energy, given by Eq. (7), as

$W_{a}^{S}=\frac{R T}{N \cdot a} \ln \left(\frac{V n}{V_{n_{\text {ref }}}}\right)$

By carrying out experiments at different temperatures, it was possible to determine the enthalpy of adsorption and the entropy of adsorption, respectively $\Delta H$ and $\Delta S$, from plots of $\Delta G / T$ versus $1 / T$, (Fig. 6 ), according to the following equation:

$\frac{\Delta G}{T}=\frac{\Delta H}{T}-\Delta S$

The acidic and basic constants, respectively $K_{a}$ and $K_{b}$, were calculated from the plot of $-\Delta H^{s} / A N^{*}$ versus $D N / A N^{*}$, according to Eq (10) [26] (Fig. 8).

$\frac{\left(-\Delta H^{s}\right)}{A N *}=K_{a} \frac{D N}{A N *}+K_{b}$

Here, $A N^{*}$ and $D N$ are, respectively, the Gutmann's modified acceptor and donor numbers of the probes tested [25,29] (Table 2).

\subsubsection{IGC experimental set-up}

In experimental work involving IGC, conventional GC equipment is generally used, with some adaptations [15].

The instrument used was a Fisons GC9100 unit (Fisons Scientific Equipment Ltd., Loughborough, UK), equipped with a FID detector The marker probe used was methane. Typically, the syringe was filled with $0.1 \mu$ l of each probe, flushed 10 times, in order to ensure the creation of a Henry's infinite dilution region, and injected manually. The injector was heated to $150{ }^{\circ} \mathrm{C}$ and the FID detector to $180^{\circ} \mathrm{C}$. The attenuation was set to 1 . The flow rate was controlled using a needle valve pressure regulator and determined using a bubble flow metre that was equipped with a helium trap [33] and thermometer. The inlet pressure, $P_{i}$, was measured using a pressure gauge and the atmospheric pressure, $P_{0}$, was obtained through the British Atmospheric Data Centre (www.badc.rl.ac.uk). The IGC unit was kept on continuously during the entire course of the work. The temperature was increased incrementally.

Due to the lack of an appropriate solvent, instead of coating a support material, the polymer was used as received, after grinding and sieving to achieve an appropriate particle size. To this end, the polymer particles were processed in a cryogenic grinder while being cooled with liquid nitrogen, followed by sieving the material through $125 \mu \mathrm{m}$ and $250 \mu \mathrm{m}$ filter gauzes and the column filled. The sieving operation excluded fine particles that would increase undesirably the pressure drop in the column.

\subsection{Differential scanning calorimetry}

The instrument used was a DSC 2010 DSCalorimeter. All the studies were carried out under a nitrogen flow rate of $200 \mathrm{~cm}^{3} / \mathrm{min}$. The typical sample mass was 3-9 mg. For the heating mode analysis, the samples were heated at a rate of $10^{\circ} \mathrm{C} / \mathrm{min}$, from $50^{\circ} \mathrm{C}$ to $440^{\circ} \mathrm{C}$. The non-isothermal crystallisation behaviour of tapeextruded blends was studied using the following temperature programme, (i) heating the sample from room temperature to 


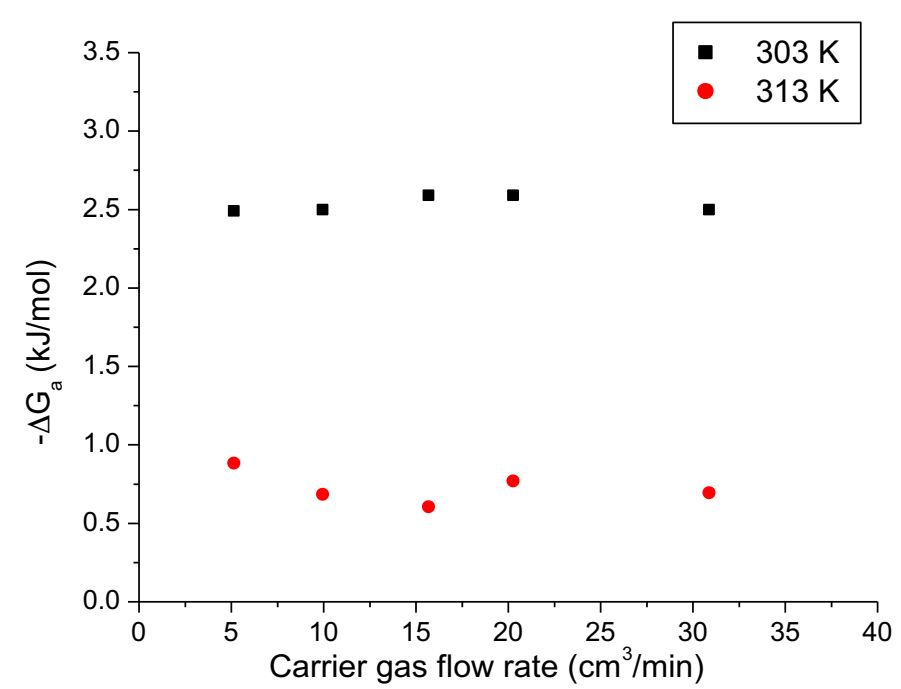

Fig. 2. Influence of the carrier gas flow rate on the energy of adsorption of n-decane on the surface of PBT B.

$250^{\circ} \mathrm{C}$ at $200^{\circ} \mathrm{C} / \mathrm{min}$, (ii) keeping the sample at this temperature for one minute, to release all the stresses within the material and to erase the thermal history and (iii) cooling the sample from $250^{\circ} \mathrm{C}$ at a constant rate $\left(8^{\circ} \mathrm{C} / \mathrm{min}\right)$ to $162^{\circ} \mathrm{C}$. In practice, the sample was held at $250^{\circ} \mathrm{C}$ for $2 \mathrm{~min}$ and $40 \mathrm{~s}$, due to the temperature equilibration stage that was prior to the isothermal step. The non-isothermal crystallisation properties that were determined were the crystallisation temperature, $T_{c}$, and the enthalpy of crystallisation, $-\Delta H_{c}$.

\section{Results and discussion}

\subsection{Inverse gas chromatography}

Carrier gas flow rates, ranging from $3 \mathrm{~cm}^{3} / \mathrm{min}$ to $35 \mathrm{~cm}^{3} / \mathrm{min}$, were used to assess the influence of the flow rate on the retention times of n-decane, at $303 \mathrm{~K}$ and $313 \mathrm{~K}$, on the surface of PBT B. This preliminary study is an essential pre-requisite in surface thermodynamic characterisation by IGC, in order to ensure that surface adsorption, and not bulk absorption, is the dominant phenomenon. The results are presented in Fig. 2.

Fig. 2 indicates that the carrier gas flow rate does not influence the adsorption energy of n-decane to a significant extent. Thus, it can be concluded that surface adsorption is the dominant mechanism, and absorption of the probe molecules into the bulk of the polymer can be neglected, if temperatures lower than the $T_{g}$ are used, for the flow rate range studied. Thus, the measurement temperature used in the study of the surface PBT was varied between $298 \mathrm{~K}$ and $318 \mathrm{~K}$, in increments of $5 \mathrm{~K}$, under a carrier gas flow rate of $10 \mathrm{~cm}^{3} / \mathrm{min}$.

In order to further confirm that surface adsorption is the governing mechanism of interactions between the polymer and the probes, and that the experimental conditions chosen in this study (flow rate and temperature) are suitable for surface characterisation, a retention diagram was developed (Fig. 3) by plotting $\operatorname{Ln}\left(V_{g}\right)$ as a function of $1 / T$ [34].

In Fig. 3, no thermal changes, due to the change in the morphology of the semi-crystalline polymer as temperature increases, are observed. The linear relationships observed are an indication of the establishment of the equilibrium between the probes and the surface of PBT due to the homogeneity of the surface. It can thus be concluded that surface adsorption is the governing phenomenon.

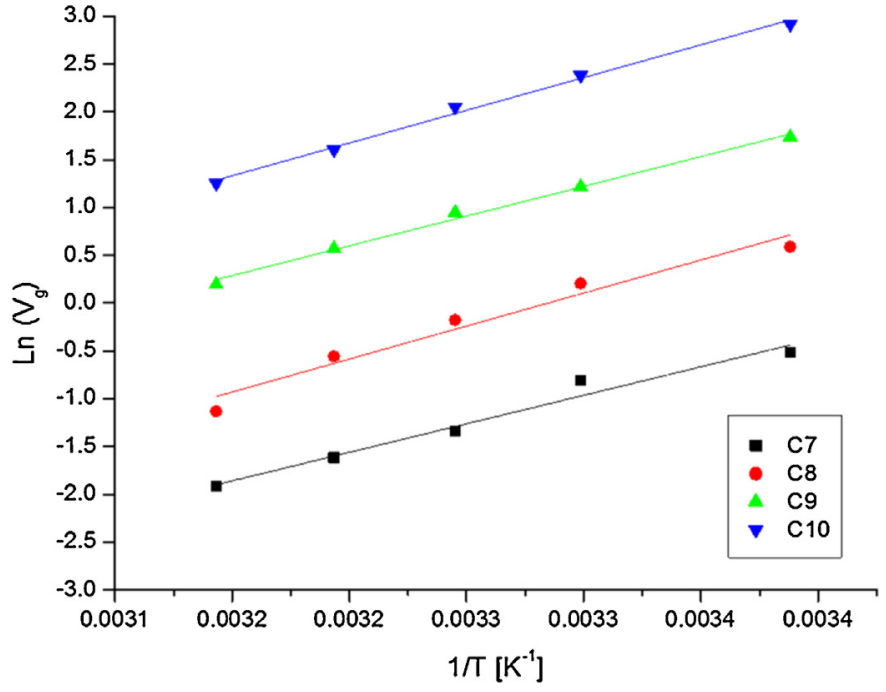

Fig. 3. Retention diagram for the apolar probes on the surface of PBT B.

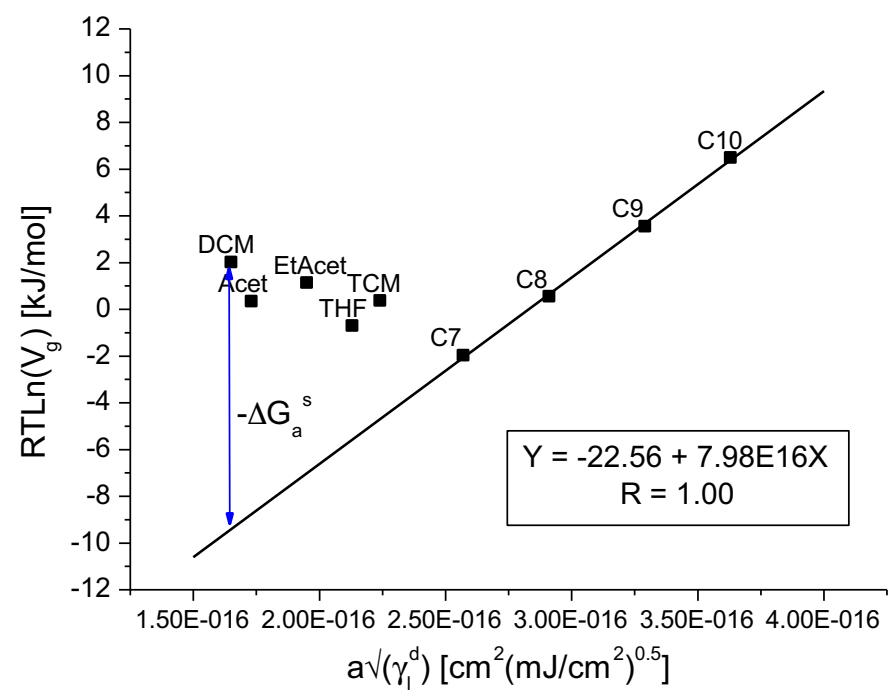

Fig. 4. Surface free energy of adsorption versus $a \sqrt{\gamma_{l}^{d}}$, for the surface adsorption of n-alkanes, and polar probes, on PBT A, at $295 \mathrm{~K}$.

\subsubsection{Dispersive component of the surface free energy of PBT A and of PBT B}

Fig. 4 illustrates the determination of the dispersive component of the surface tension of PBT A, at $295 \mathrm{~K}$, according to Fowkes' approach.

Table 3 summarises the values determined for the dispersive component of the surface tension of PBT A and of PBT B. The values presented indicate that the dispersive component of the surface tension remains constant, within experimental error, for the

Table 3

Values of the dispersive component of the surface tension of PBT A and of PBT B.

\begin{tabular}{llllll}
\hline & \multicolumn{2}{l}{ PBT A } & & \multicolumn{2}{l}{ PBT B } \\
\cline { 2 - 3 } \cline { 5 - 6 }$T(\mathrm{~K})$ & $\gamma_{s}^{d}\left(\mathrm{~mJ} / \mathrm{m}^{2}\right)$ & $R^{2}$ & & $\gamma_{s}^{d}\left(\mathrm{~mJ} / \mathrm{m}^{2}\right)$ & $R^{2}$ \\
\hline 295 & $43.9 \pm 2.1$ & 1.00 & & $42.4 \pm 1.0$ & 1.00 \\
303 & $40.7 \pm 1.9$ & 0.98 & & $42.5 \pm 2.8$ & 0.99 \\
308 & $42.8 \pm 2.4$ & 1.00 & & $41.4 \pm 2.6$ & 1.00 \\
313 & $40.0 \pm 0.6$ & 1.00 & & $40.7 \pm 1.3$ & 1.00 \\
318 & $40.7 \pm 2.1$ & 1.00 & & $44.7 \pm 9.9$ & 0.92 \\
Average & $41.6 \pm 1.7$ & n/a & $42.3 \pm 1.5$ & $\mathrm{n} / \mathrm{a}$ \\
\hline
\end{tabular}




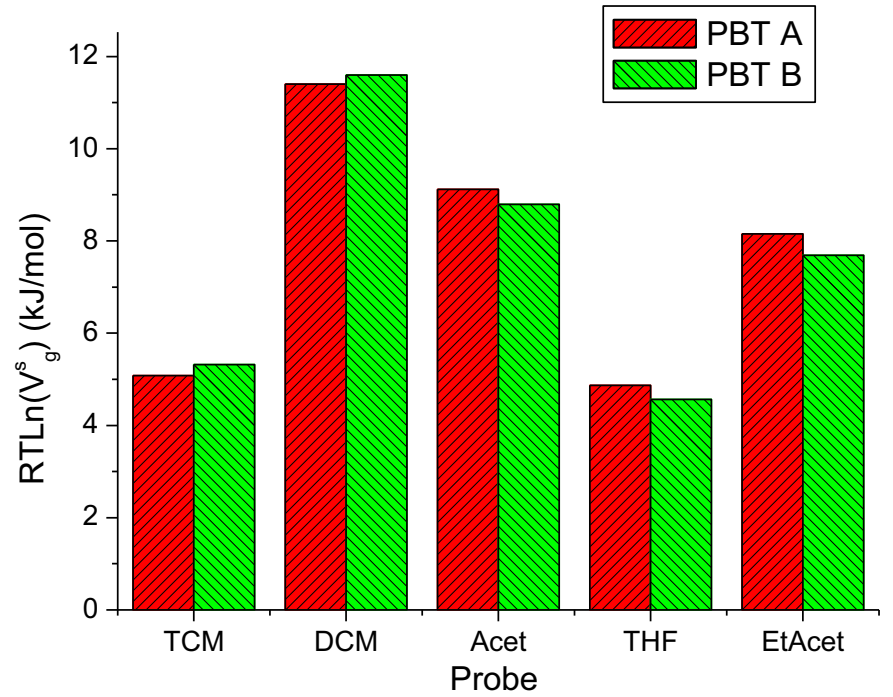

Fig. 5. Comparison of the specific component values of the energy of adsorption of the polar probes on the surface of PBT A with those relating to the surface of PBT B, at $295 \mathrm{~K}$.

temperature range studied, and equal is to $41.6 \pm 1.7 \mathrm{~mJ} / \mathrm{m}^{2}$ and $42.3 \pm 1.5 \mathrm{~mJ} / \mathrm{m}^{2}$, for PBT A and PBT B, respectively. The values of $\gamma_{s}^{d}$ for PBT A and for PBT B are practically identical. This leads to the conclusion that the different end-group composition (Table 1) does not influence to a noticeable extent, the dispersive component of the surface tension. Bearing in mind that the major difference between PBT A and PBT B is in the $\mathrm{OH}$ end-group concentration, the value found for $\gamma_{s}^{d}$ confirms the observations found in literature [35] with regard to the fact that this functional group, as a high-energy site, contributes mainly to the formation of specific intermolecular interactions. The values found are in accord with surface tension studies on PBT based on contact angle measurements [23].

\subsubsection{Adsorption of polar probes on $P B T A$ and on $P B T B$}

In Fig. 5 are compared the values of $-\Delta G_{a}^{S}$ for the polar probes on the surface of PBT A with those concerning the surface of PBT B, at $295 \mathrm{~K}$. The retention times for DEE on the surface of PBT A were very low and, therefore, prone to large experimental errors. Consequently, the determination of the energy of adsorption, and of the specific component of the enthalpy of adsorption of DEE, was not possible. This low retention time probably results from steric hindrance, that is, structural restrictions, from both the adsorbate and the adsorbent, hindering these molecules from spatial conformations becoming effective in terms of specific intermolecular interactions. The oxygen atom (Lewis basic centre) of diethyl ether (DEE) is more susceptible to shielding by the neighbouring hydrogen atoms [36] than is that of, e.g. acetone (Acet), which is readily accessible for interaction. Similar results have been observed when $\mathrm{CCl}_{4}$ was used as a probe [37].

For both PBT A and PBT B, the adsorption of the Lewis acidic probes is as strong as, or stronger than, the adsorption of Lewis basic/Lewis amphoteric probes. Bearing in mind the relatively low acidity of the acidic probes, when compared to the basicity of the basic probes (e.g. THF), it can be concluded that the surfaces of both PBT A and PBT B are amphoteric, being strongly Lewis basic. Fig. 5 also shows that the surface of PBT A is comparable to that of PBT B in terms of Lewis acidity/basicity. An analysis of the specific component of the enthalpy of adsorption will provide a better insight into the differences between the Lewis acidic/basic properties of the two PBTs. The values of the enthalpy, and entropy, of adsorption of the polar probes, along with the corresponding dispersive and
Table 4

Dispersive components of the enthalpy of adsorption and of the entropy of adsorption, $\Delta H^{d}{ }_{a}$, and $\Delta S^{d}{ }_{a}$, respectively, of the polar probes, on the surface of PBT A

\begin{tabular}{llll}
\hline Probe molecule & $-\Delta H^{d}{ }_{a}(\mathrm{~kJ} / \mathrm{mol})$ & $\Delta S^{d}{ }_{a}(\mathrm{~J} / \mathrm{mol} \mathrm{K})$ & $R^{2}$ \\
\hline TCM & 33.0 & -127.0 & 0.91 \\
DCM & 26.2 & -120.0 & 0.81 \\
Acet & 27.1 & -121.0 & 0.83 \\
THF & 31.8 & -126.0 & 0.89 \\
EtAcet & 29.7 & -124.0 & 0.87 \\
\hline
\end{tabular}

\section{Table 5}

Specific components of the enthalpy of adsorption and of the entropy of adsorption $\Delta H^{s}{ }_{a}$ and $\Delta S^{d}{ }_{a}, \Delta S^{s}{ }_{a}$, respectively, of the polar probes, on the surface of PBT A

\begin{tabular}{llll}
\hline Probe molecule & $-\Delta H^{s}{ }_{a}(\mathrm{~kJ} / \mathrm{mol})$ & $\Delta S^{s}{ }_{a}(\mathrm{~J} / \mathrm{mol} \mathrm{K})$ & $R^{2}$ \\
\hline TCM & -38.4 & 147.0 & 0.94 \\
DCM & -23.1 & 138.0 & 0.82 \\
Acet & -20.9 & 101.0 & 0.89 \\
THF & -23.0 & 93.7 & 0.81 \\
EtAcet & -17.2 & 85.8 & 0.92 \\
\hline
\end{tabular}

specific components, are summarised in Tables 4 and 5 for PBT A, and their determination is illustrated in Fig. 6 for this polymer. The values relating to PBT B have already been reported in a previous publication [22].

The specific component of the enthalpy of adsorption of polar probes on the surface of PBT A is endothermic. This confirms the reported thermodynamic analysis of the surface and bulk PBT B [22]. Endothermic values of energy of adsorption have also been reported in the literature for the adsorption of polar probe molecules on titanium dioxide pigments [37,38], on a vinyl acetate-vinyl alcohol copolymer [39], and on 2(N-morpholino)ethylmethacrylate [40]. A rearrangement of the surface upon the chemisorption of polar molecules is thought to occur, thus initiating an increase in the entropy of the system. This rationale is confirmed by the positive values of $\Delta S^{s}$, and supported by the experimentally verified phenomenon of the rearrangement of the surface layers due to chemisorption [41].

The enthalpy of formation of individual hydrogen bonds is always negative. However, the total enthalpy (and entropy) related to the formation of hydrogen bonds is the result of three contributions [42]: a positive contribution that results from breaking hydrogen bonds in the self-associating polymer, a negative contribution that results from forming hydrogen bonds between the self-associating polymer and the adsorbent molecule, and contributions from van der Waals and dipole forces. When polar molecules

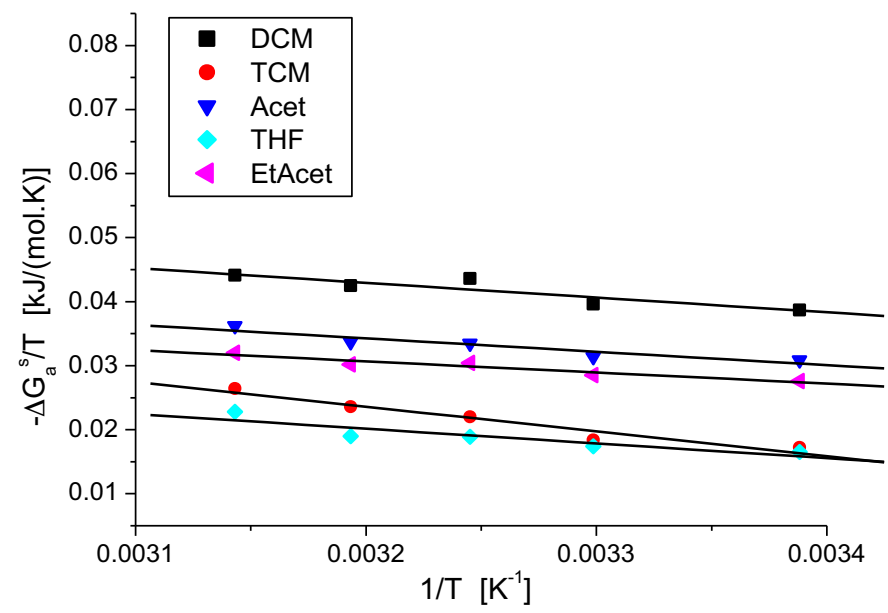

Fig. 6. Determination of the specific component of the enthalpy and of the entropy of adsorption, of polar probes on the surface of PBT A. 


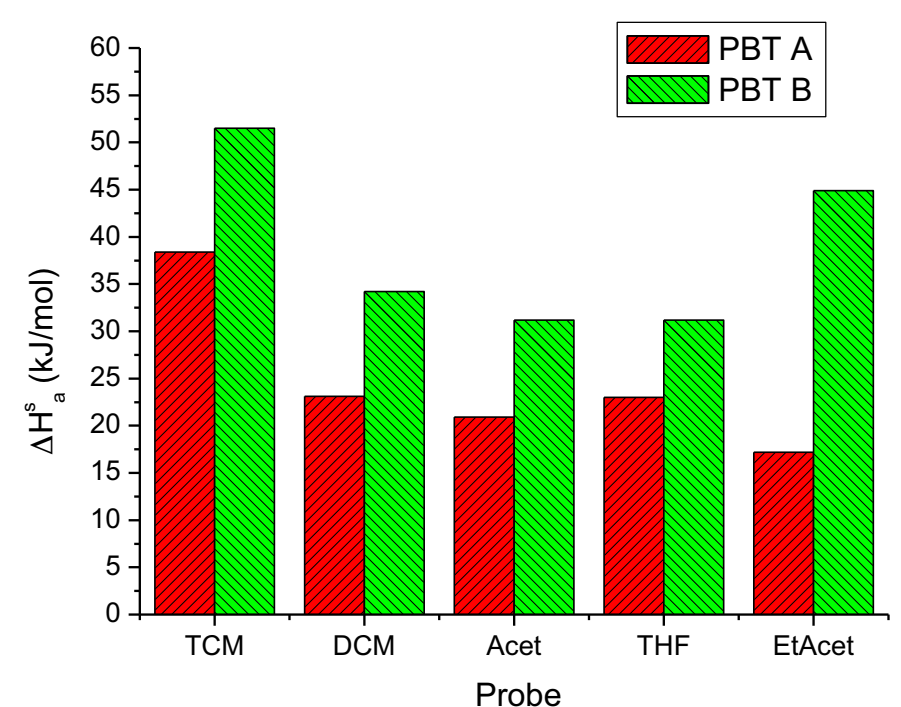

Fig. 7. Comparison of the specific component values of the enthalpy of adsorption of the polar probes on the surface of PBT A with those relating to the surface of PBT B.

Table 6

Values of $K_{a}$ and $K_{b}$ determined for the surfaces of PBT A and PBT B.

\begin{tabular}{llll}
\hline & $K_{a}$ & $K_{b}$ & $R^{2}$ \\
\hline PBT A & -0.24 & -0.97 & 0.97 \\
PBT B & -0.49 & -0.96 & 0.96 \\
\hline
\end{tabular}

adsorb on PBT, the dominant factor is the positive contribution arising from the breaking of hydrogen bonds in the self-associating polymer. This results in an endothermic enthalpy of adsorption and an increase in the entropy of the system [22].

It should be noticed (Tables 4 and 5 ) that the dispersive component of the enthalpy of adsorption, $\Delta H^{d}{ }_{a}$, is exothermic, that the corresponding change in entropy, $\Delta S^{d}$, negative, and that the values are similar for most of the polar probes. Furthermore, the value of $-\Delta H^{d}{ }_{a}$ increases with increasing $a \times \sqrt{\gamma_{l}^{d}}$.

In the case of PBT A, an analysis of the specific component of the enthalpy of adsorption, leads to the following ranking (the more negative, the stronger the interaction, as it is endothermic): $\mathrm{TCM}>\mathrm{DCM}=\mathrm{THF}>$ Acet $>$ EtAcet. This indicates that PBT A is amphoteric and $K_{b}$ ought to be high, in line the analysis of the specific component of the energy of adsorption of the polar probes. The change in entropy upon adsorption is greater for those probes whose adsorption is characterised by the greater specific component of the enthalpy of adsorption. The analysis of $-\Delta H^{s} a$ for PBT $A$ is also in line with the analysis of the energy of adsorption, and with the results relating to the surface and bulk PBT B [22].

Fig. 7 gives a comparison of the values of $\Delta H^{s}{ }_{a}$ of the polar probes on the surface of PBT A with those concerning the surface of PBT B.

Fig. 7 suggests that the surface of PBT A should be less Lewis acidic and less Lewis basic, than that of PBT B. The determination of $K_{a}$ and of $K_{b}$ provides a more complete description of the surface Lewis acidity/basicity.

\subsubsection{Determination of $K_{a}$ and $K_{b}$ for the surface of PBT $A$ and for the surface of PBT B}

The determination of the surface Lewis acidity constant, $K_{a}$, and of the surface Lewis basicity constant, $K_{b}$, is illustrated in Fig. 8 for PBT A. The values obtained for both PBT A and PBT B are summarised in Table 6.

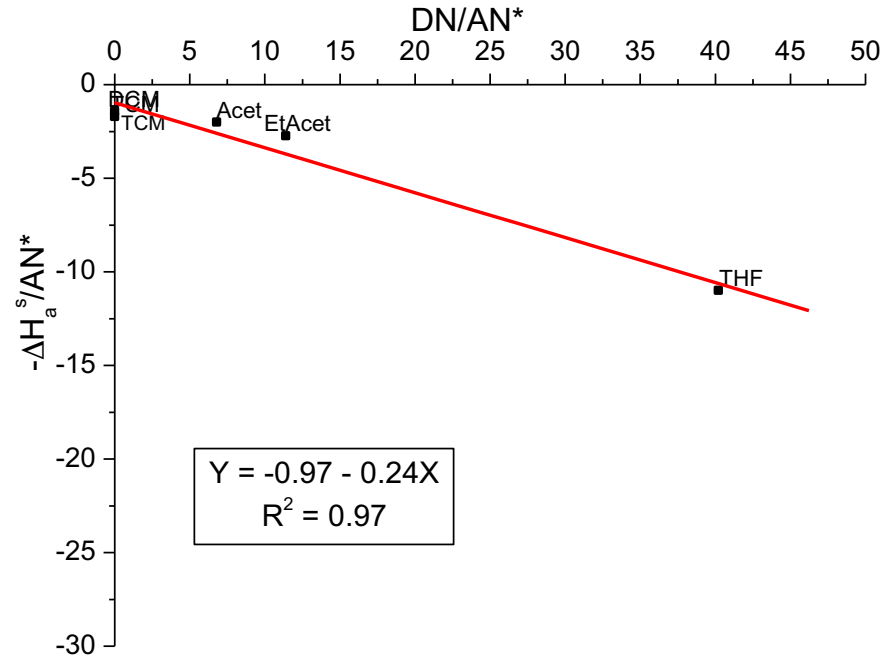

Fig. 8. Determination of the $K_{a}$ and of the $K_{b}$ of the surface of PBT A.

It should be noted that the $K_{a}$ and $K_{b}$ values are negative due to the endothermic adsorption of the probe molecules. The surfaces of PBT A and PBT B are concluded to be Lewis amphoteric and strongly Lewis basic, in line with the analysis of the energy and enthalpy of adsorption of polar probes. The values determined for $K_{a}$ and $K_{b}$ are consistent with the analysis of the structure of this molecule (Fig. 1). Thus, the Lewis acidic sites have their origin in the hydrogen atoms of the $-\mathrm{O}-\mathrm{CH}_{2}$ - segments, and in the hydrogen atoms of the hydroxyl end-group and of the carboxylic end-group. The Lewis basic sites have their origins in the ester moiety, and in the oxygen atoms of the carboxylic end-group and of the hydroxyl end-group [43].

The poly(butylene terephthalate), PBT A, is significantly less Lewis acidic than PBT B and as Lewis basic as PBT B. This can be rationalised on the basis of an analysis of the carboxyl end-group and hydroxyl end-group concentrations in both PBT A and PBT B, as determined by FTIR (Table 1). The hydroxyl end-group concentration of PBT A is lower than that of PBT B, which is reflected in the lower acidic character of PBT A. The carboxyl end-group concentration differences between PBT A and PBT B do not influence significantly the Lewis basicity, as quantified by $K_{b}$. This observation arises from the dominant contribution of the ester functionality to the surface basicity, alongside with the contribution of the basic oxygen atom in the hydroxyl groups. The fact that the $K_{b}$ value for the two PBTs does not fully correlate with the value of the specific component of the enthalpy of adsorption of DCM and of TCM may be related to the relatively low value of $A N^{*}$ for DCM and TCM, when compared to the value of $\mathrm{DN}$ for the remaining polar probes. This result stresses (i) the importance of using as many polar probes as possible in the determination of $K_{a}$ and $K_{b}$, and (ii) the need for a systematic and holistic analysis of $\Delta G^{S}{ }_{a},-\Delta H^{s} a, K_{a}$ and $K_{b}$, in contrast to an individual analysis of these thermodynamic parameters (as is usually the case in the IGC literature).

\subsection{Study of the crystallisation properties of PBT A and PBT B}

The values of $K_{a}$ and $K_{b}$ for both PBT A and PBT B indicate that the intramolecular interactions and the intermolecular interactions between PBT molecules are strong. This molecule is characterised by the non-existence of bulky side-groups, by the chemically regular structure, and by high mobility (due to the butylene unit in the chain). Thus, both the thermodynamic and the structural requirements for crystallinity exist in the case of PBT molecules. 


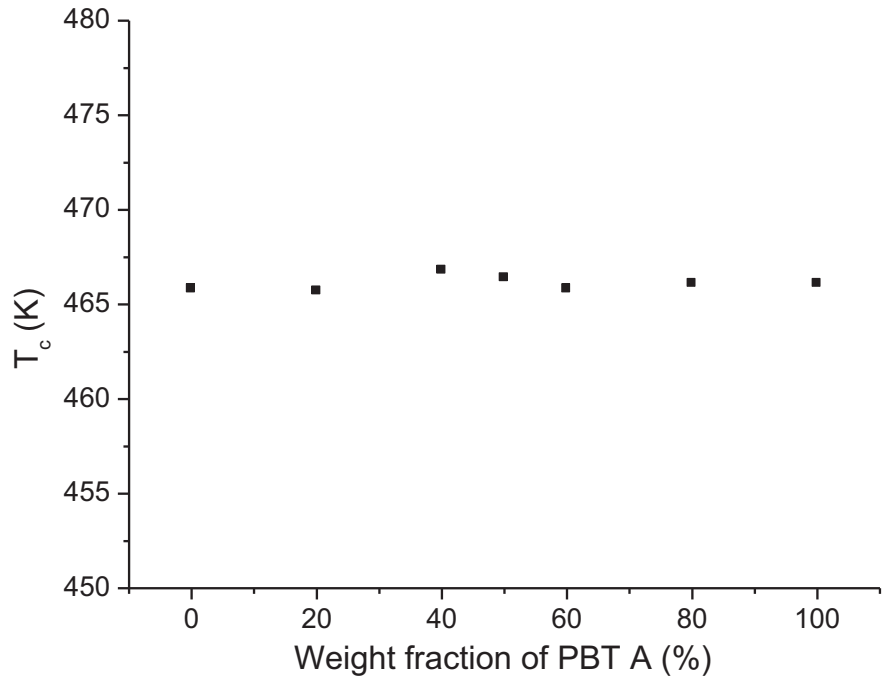

Fig. 9. Non-isothermal crystallisation temperature versus PBT A loading in samples of PBT granulate.

To assess the influence of the end-group type and concentration, and of molecular weight differences on the crystallisation properties of PBT, a series of PBT A plus PBT B granulate samples was prepared with increasing amounts of PBT A. The values determined for the cold crystallisation temperature, $T_{c}$, and for the enthalpy of crystallisation, $-\Delta H_{c}$, of these samples are presented in Figs. 9 and 10, respectively.

The crystallinity degree of PBT A is calculated as $56 \%$ and that of PBT B as $65 \%$ (considering the value of $142 \mathrm{~J} / \mathrm{g}$ for the $-\Delta H_{c}$ of $100 \%$ crystalline PBT). The extent of crystallisation decreases linearly with increasing amount of PBT A in the PBT granulate sample. The greater extent of crystallinity of PBT B is interpreted, in view of the values determined for $K_{a}$ and $K_{b}$, to be a consequence of greater strength of specific Lewis acid/base intermolecular interactions than in PBT A. This is in accordance with the fact that high energy sites in polymeric materials (as assessed by IGC at infinite dilution) are well known to act as nucleating spots that initiate the crystallisation process $[44,45]$. The differences in the average molecular weight of PBT A and PBT B also play a role in the crystallisation properties of this polymer. Lower molecular weight values would be expected to lead to greater values of crystallinity degree. However, in this study it was not possible to distinguish the

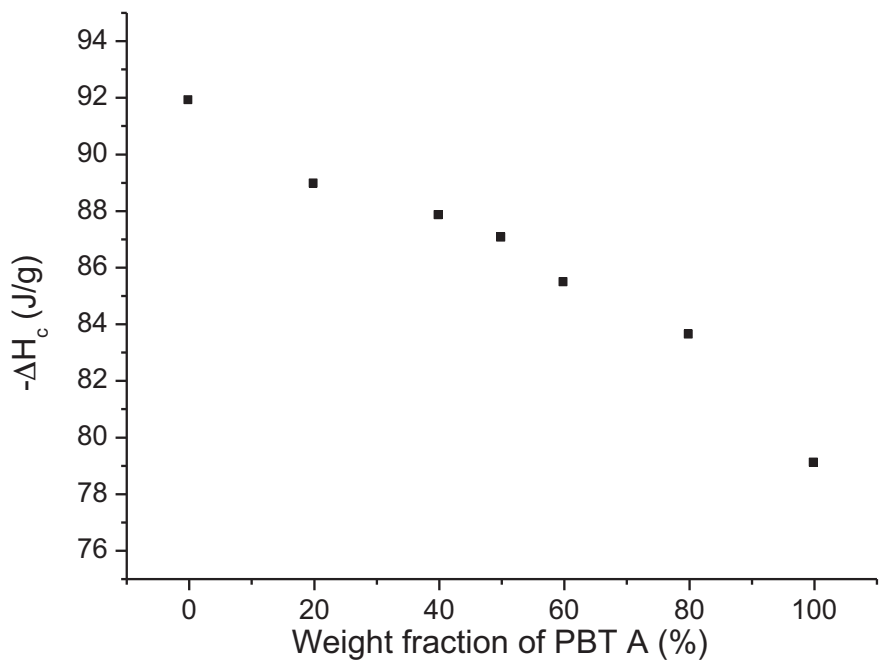

Fig. 10. Enthalpy of crystallisation versus PBT A loading in samples of PBT granulate. relative contribution of molecular weight differences and of endgroup type and concentration differences. This is due to the fact that the greater $-\mathrm{OH}$ end-group concentration is directly related with the lower average molecular weight of this polymer.

Fig. 9 also shows, very interestingly, that the non-isothermal crystallisation temperature is not significantly influenced by the weight fraction of PBT A in the sample. Thus, differences in the end-group type and concentration and in the average molecular weight of these PBTs do not cause changes in the crystallisation activation energy. In view of the values found for $K_{a}$ and $K_{b}$, one can argue that the crystallisation activation energy is, in this case, dominantly defined by the energy of the Lewis basic sites. This observation derives from the significantly greater value of $K_{b}$ when compared to the value of $K_{a}$, for both PBT A and PBT B, and from the similar value of $K_{b}$ for these grades of PBT.

\section{Conclusions}

The ability of PBT to interact through dispersive intermolecular forces is not influenced by the end-group type and concentration or by the molecular weight. The enthalpy of adsorption of polar molecules on PBT was confirmed to be endothermic. This result was interpreted as the dominant contribution to the enthalpy and entropy of adsorption of breaking hydrogen bonds in the polymer, compared with the contribution of forming hydrogen bonds between the polar probe molecules and the PBT molecules.

IGC characterisation of the surface Lewis acidic/basic properties of PBT A and of PBT B correlates well with the carboxyl end-group concentration and hydroxyl end-group concentration, as determined by FTIR. Furthermore, the analysis of the surface Lewis acidity and surface Lewis basicity, as quantified by $K_{a}$ and $K_{b}$ and determined by IGC, alongside with the analysis of the physical structure of these polymers, is useful in the interpretation of the crystallinity and, thus, of the excellent solvent resistance and thermal stability of PBT.

The differences in the end-group type and concentration, and in the average molecular weight, between PBT A and PBT B, do not cause differences in the crystallisation activation energy, and thus, in the crystallisation temperature. This evidence is interpreted in terms of dominant contribution of the Lewis basic sites to the crystallisation activation energy. The extent of crystallisation is lower for PBT A than it is for PBT B. This is a consequence of weaker Lewis acid/base intramolecular and intermolecular interactions and of greater molecular weight in the case of PBT A.

\section{Acknowledgements}

The authors wish to acknowledge the support and the collaboration of SABIC Innovative Plastics, Bergen op Zoom, The Netherlands, in this project.

\section{References}

[1] V.M. Nadkarni, J.P. Jog, Crystallization behaviour in polymer blends, in: L.A. Utracki (Ed.), Two-Phase Polymer Systems, Munich, Germany, 1991, pp. 213-239.

[2] C.C. Huang, F.-C. Chang, Reactive compatibilization of polymer blends of poly(butylene terephthalate) (PBT) and polyamide-6,6 (PA66): 1. Rheologica and thermal properties, Polymer 38 (1997) 2135-2141.

[3] S.P. Mishra, P. Venkidusamy, Structural and thermal behavior of PC/PBT blends, J. Appl. Polym. Sci. 58 (1995) 2229-2234.

[4] Y.-Y. Cheng, M. Brillhart, P. Cebe, M. Capel, X-ray scattering and thermal analysis study of the effects of molecular weight on phase structure in blends of poly(butylene terephthalate) with polycarbonate, J. Polym. Sci. B: Polym. Phys. 34 (1996) 2953-2965.

[5] I. Pillin, S. Pimbert, J.F. Feller, G. Levesque, Crystallization kinetics of poly(butylene terephthalate) (PBT): influence of additives and free carboxylic acid chain ends, Polym. Eng. Sci. 41 (2001) 178-191. 
[6] G. Pompe, L. Haubler, W. Winter, Investigations of the equilibrium melting temperature in PBT and PC/PBT blends, J. Polym. Sci. B: Polym. Phys. 34 (1996) $211-219$.

[7] G. Pompe, Influence of transesterification on the thermal properties of PC/PBT blend-miscibility of PC and PBT, Recent Res. Dev. Polym. Sci. 1 (1997) 109-147.

[8] G. Pompe, E. Meyer, H. Komber, H. Hamann, Influence of PBT crystallization on miscibility degree of amorphous phase in PC/PBT melt blends, Thermochim. Acta 187 (1991) 185-200.

[9] M.E.J. Dekkers, S.Y. Hobbs, I. Bruker, V.H. Watkins, Migration of polymer blend components during melt compounding, Polym. Eng. Sci. 30 (1990) 1628-1632.

[10] M. Ratzsch, G. Haudel, G. Pompe, E. Meyer, Interaction between polymers, J. Macromol. Sci. A Chem. A 27 (1990) 1631-1655.

[11] H. Bertilsson, B. Franzen, J. Kubat, Ageing of PC/PBT blends. II: Phase separation effects, Plastics Rubber Process Appl. 10 (1988) 145-153.

[12] A.N. Wilkinson, S.B. Tattum, A.J. Ryan, Melting, reaction and recrystallization in a reactive PC-PBT blend, Polymer 38 (1997) 1923-1928.

[13] G. Pompe, L. Haubler, Investigations of transesterification in PC/PBT melt blends and the proof of immiscibility of PC and PBT at completely suppressed transesterification, J. Polym. Sci. B Polym. Phys. 35 (1997) 2161-2168.

[14] D.G. Hamilton, R.R. Gallucci, The effects of molecular weight on polycarbonate-polybutylene terephthalate blends, J. Appl. Polym. Sci. 48 (1993) 2249-2252.

[15] J.M.R.C.A. Santos, J.T. Guthrie, Analysis of interactions in multicomponent polymeric systems: the key-role of inverse gas chromatography, Mater. Sci. Eng. R: Rep. 50 (2005) 79-107.

[16] A. Voelkel, Physicochemical measurements (inverse gas chromatography), in: C. Poole (Ed.), Gas Chromatography, Elsevier, Amsterdam (The Netherlands), 2012, pp. 477-494.

[17] A. Voelkel, B. Strzemiecka, K. Adamska, K. Milczewska, Inverse gas chromatography as a source of physiochemical data, J. Chromatogr. A 1216 (2009) $1551-1566$

[18] A. Voelkel, K. Adamska, Properties of materials as determined by inverse gas chromatography, Ann. UMCS Chem. 64 (2009) 169-183.

[19] L.H. Lee, Relevance of the density-functional theory to acid-base interactions and adhesion in solids, in: K.L. Mittal, J. Anderson (Eds.), Acid-Base Interactions: Relevance to Adhesion Science and Technology, VSP, Utrecht, The Netherlands, 1991, pp. 25-46.

[20] P. Mukhopadhyay, H.P. Schreiber, Inverse gas chromatography for polymer surface characterization above and below Tg, Macromolecules 26 (1993) 6391-6396.

[21] J. Schultz, L. Lavielle, Interfacial properties of carbon fiber-epoxy, in: D.R. Lloyd, T.C. Ward, H.P. Schreiber (Eds.), Inverse Gas Chromatography, Characterization of Polymers and Other Materials, A.C.S., Washington, 1989, pp. 185-202.

[22] J.M.R.C.A. Santos, K. Fagelman, J.T. Guthrie, Characterisation of the surface Lewis acid-base properties of poly (butylene terephthalate) by inverse gas chromatography, J. Chromatogr. A 969 (2002) 111-118.

[23] S.Y. Hobbs, M.E.J. Dekkers, V.H. Watkins, Effect of interfacial forces on polymer blend morphologies, Polymer 29 (1988) 1598-1602.

[24] S. Mohammadi-Jam, K.E. Waters, Inverse gas chromatography applications: a review, Adv. Colloid Interface Sci. 212 (2014) 21-44.

[25] D.P. Kamdem, S.K. Bose, P. Luner, Inverse gas chromatography characterization of birch wood meal, Langmuir 9 (1993) 3039-3044.
[26] H. Chtourou, B. Riedl, B.V. Kokta, Surface characterizations of modified polyethylene pulp and wood pulps fibers using XPS and inverse gas chromatography, J. Adhesion Sci. Technol. 9 (1995) 551-574.

[27] J.M.R.C.A. Santos, M.H. Gil, A. Portugal, J.T. Guthrie, Characterisation of the surface of a cellulosic multi-purpose office paper by inverse gas chromatography, Cellulose 8 (2001) 217-224.

[28] J.M.R.C.A. Santos, K. Fagelman, J.T. Guthrie, Characterisation of the surface Lewis acid-base properties of the components of pigmented, impact-modified, bisphenol A polycarbonate-poly(butylene terephthalate) blends by inverse gas chromatography-phase separation and phase preferences, J. Chromatogr. A 969 (2002) 119-132.

[29] D.P. Kamdem, B. Riedl, Inverse gas chromatography of lignocellulosic fibers coated with a thermosetting polymer: use of peak maximum and conder and young methods, J. Colloid Interface Sci. 150 (1992) 507-516.

[30] P.L. Jackson, M.B. Huglin, A. Cervenka, Use of inverse gas chromatography to quantify interactions in amine cured epoxy resins, Polym. Int. 35 (1994) $119-133$.

[31] F.M. Fowkes, J.A. Manson, T.B. Lloyd, D.O. Tischler, B.A. Shah, Inverse gas chromatography in: D.M. Mattox (Ed.), Mater. Res. Soc. Symp., vol. 119, 1988, pp. 223-234.

[32] J. Riddle, F.M. Fowkes, Spectral shifts in acid-base chemistry. 1. Van der Waals contributions to acceptor numbers, J. Am. Chem. Soc. 112 (1990) 3259-3264.

[33] A.E. Bolvari, et al., Experimental techniques in IGC, in: D.R. Lloyd, T.C. Ward, H.P. Schreiber (Eds.), Inverse Gas Chromatography, Characterization of Polymers and Other Materials, A.C.S., Washington, 1989, pp. 12-19.

[34] M. Kunaver, et al., Inverse gas chromatography-a different approach to characterization of solids and liquids, Acta Chim. Slov. 51 (2004) 373-394.

[35] W. Zhang, A.I. Leonov, IGC study of filler-filler and filler-rubber interactions in silica-filled compounds, J. Appl. Polym. Sci. 81 (2000) 2517-2530.

[36] A.C. Tiburcio, J.A. Manson, Acid-base interactions in filler characterization by inverse gas chromatography, J. Appl. Polym. Sci. 42 (1991) 427-438.

[37] C.R. Hegedus, I.L. Kamel, Thermodynamic analysis of pigment and polymer surfaces using inverse gas chromatography, J. Coat. Technol. 65 (1993) $31-43$

[38] Y.J. Lee, I. Manas-Zloczower, D.L. Feke, Characterization of solvent interactions with treated titanium dioxide powders, Powder Technol. 73 (1992) 139-146.

[39] F. Chen, Study of acceptor-donor interactions at the polymer interface by inverse gas chromatography data analysis, Macromolecules 21 (1988) $1640-1643$.

[40] A. Askin, D.T. Yazıcı, A study of the surface analysis of some water-soluble polymers by inverse gas chromatography, Surf. Interface Anal. 40 (2008) 1237-1241.

[41] V. Ponec, Z. Knor, S. Cerny, Adsorption on Solids, Butterworths, London, 1974.

[42] M.M. Coleman, J.F. Graf, P.C. Painter, Specific Interactions and the Miscibility of Polymer Blends, Technomic Pub. Co., Lancaster, PA, U.S.A., 1991.

[43] P.L. Huyskens, W.A.P. Luck, T. Zeegers-Huyskens, Intermolecular Forces, An Introduction to Modern Methods and Results, Springer-Verlag, London, 1991.

[44] M. Soleimani, L. Tabil, S. Panigrahi, I. Oguocha, Crystallization and thermal properties of biofiber-polypropylene composites, in: El-Sonbati Adel (Ed.), Thermoplastic - Composite Materials, InTech, Croatia, 2012, pp. 131-146.

[45] D. Page, T. Gopakumar, Properties and crystallization of maleated polypropylene/graphite flake nanocomposites, Polym. J. 38 (2006) 920-929. 